\title{
Frequency scanning microstrip antennas
}

\section{Danielsen, Magnus; Jørgensen, Rolf}

\section{Published in:}

I E E E Transactions on Antennas and Propagation

Publication date:

1979

\section{Document Version}

Publisher's PDF, also known as Version of record

Link back to DTU Orbit

\section{Citation (APA):}

Danielsen, M., \& Jørgensen, R. (1979). Frequency scanning microstrip antennas. I E E E Transactions on Antennas and Propagation, 27(2), 146-150.

\section{General rights}

Copyright and moral rights for the publications made accessible in the public portal are retained by the authors and/or other copyright owners and it is a condition of accessing publications that users recognise and abide by the legal requirements associated with these rights.

- Users may download and print one copy of any publication from the public portal for the purpose of private study or research.

- You may not further distribute the material or use it for any profit-making activity or commercial gain

- You may freely distribute the URL identifying the publication in the public portal

If you believe that this document breaches copyright please contact us providing details, and we will remove access to the work immediately and investigate your claim. 


\title{
Frequency Scanning Microstrip Antennas
}

\author{
MAGNUS DANIELSEN AND ROLF J $\emptyset$ RGENSEN
}

\begin{abstract}
The principles of using radiating microstrip resonators as elements in a frequency scanning antenna array are described. The resonators are cascade-coupled. This gives a scan of the main lobe due to the phase-shift in the resonator in addition to that created by the transmission line phase-shift. Experimental results in $X$-band, in good agreement with the theory, show that it is possible to scan the main lobe an angle of $\pm 30^{\circ}$ by a variation of the frequency $\pm 300 \mathrm{MHz}$, and where the $3 \mathrm{~dB}$ beamwidth is less than $10^{\circ}$. The directivity was 14.7 $\mathrm{dB}$, while the gain was $8.1 \mathrm{~dB}$. The efficiency might be improved by a trade-off between the efficiency and the scanning angle, or by using a better amplitude distribution.
\end{abstract}

\section{INTRODUCTION}

$\mathbf{T}$ HE IDEA of utilizing the radiation from microstrip resonators was proposed and published by Munson and Howell [1], [2]. Since then, microstrip antennas have been subject to different investigations for frequencies from the VHF to the $X$-band with both circular and linear polarization [3.]-[9]. Earlier works on frequency scanning have been based on the frequency dependent phase-shift between the antenna elements created by delay in a transmission line, to which the elements were loosely coupled [10]. In [11] a printed circuit grid antenna at $2 \mathrm{GHz}$ resulted in a slightly frequencydependent beam direction.

In the present work we use microstrip antenna resonators as elements in a frequency scanning array. Contrary to previous works, we have cascade-coupled the resonators. Consequently, we obtain a phase-shift due to the transmission microstrip resonator in addition to that caused by the transmission line connecting the elements, which can yield an appreciable fraction of the total phase-shift. According to these principles a method to design a frequency-scanning antenna array based on the design of a single antenna element is described.

\section{SINGLE-MICROSTRIP RESONATOR}

The single elements in the antenna array are microstrip transmission resonators with a common conducting ground plane (Fig. 1). Each resonator radiates from its two ends [9]. Radiation from an open circuit microstrip line was first treated by Lewin [12]. The radiated power from a resonator is given formally by an identical formula as found in [12]:

$$
P=\frac{1}{2 \pi} \sqrt{\frac{\mu_{0}}{\epsilon_{0}}}\left(k_{0} h\right)^{2} F_{1}^{\prime}(\epsilon)
$$

where we have normalized the current amplitude in each of the two opposite propagating waves in the resonator to $1 \mathrm{~A}$ and

Manuscript received January 19, 1978; revised July 16, 1978.

M. Danielsen is with the Electromagnetics Institute, Technical University of Denmark, DK-2800 Lyngby, Denmark.

R. J $\phi$ rgensen was with the Electromagnetics Institute, Technical University of Denmark, Lyngby, Denmark. He is now with Ticra ApS, DK-1112 Copenhagen, Denmark.
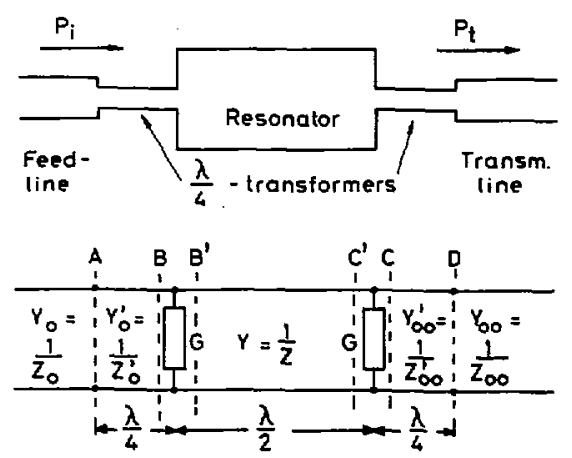

Fig. 1. Transmission microstrip resonator and its equivalent network.

neglected the coupling line. In (1), $k_{0}=2 \pi / \lambda_{0}$ is the propagation factor, $\lambda_{0}$ is the vacuum wavelength, $h$ is the strip-ground spacing, $\mu_{0}$ and $\epsilon_{0}$ are the permeability and permittivity of vacuum, respectively, $\epsilon$ is the effective relative permittivity [15], and $F_{1}{ }^{\prime}(\epsilon)=F_{1}{ }^{\prime}(\epsilon, n=1)$ a function [13] given in the Appendix. $F_{1}{ }^{\prime}(\epsilon)$ reduces to twice the function Lewin found for the open circuit, when there is no mutual coupling between the two ends [14].

In the design procedure we will use the radiation quality factor

$$
Q_{r}=\frac{\omega U}{P}
$$

where $\omega=2 \pi f$ is the cyclic frequency and

$$
U=\frac{Z}{2 f}
$$

is the normalized energy content in the resonator. $Z$ is the characteristic impedance of the resonator microstrip line.

The radiation losses are represented by two conductances placed at the ends of the resonator

$$
G=\frac{\pi}{4 Q_{r} \cdot Z} \text {. }
$$

The coupling to the element is performed by quarter wavelength transformers at the input and output. In the design, losses in the lines are neglected, but will be taken into account as restrictions to the theory. The characteristic admittances of the transformers $Y_{0}^{\prime}$ and $Y_{00}{ }^{\prime}$ and that of the resonator $Y=1 / Z$ are determined by three conditions.

1) The resonators should be matched at the input at the center frequency, giving rise to

$$
Y_{0}=Y_{\text {in }}=\frac{Y_{0}{ }^{\prime 2}}{Y_{00}{ }^{\prime 2} / Y_{00}+2 G}
$$


where $Y_{\text {in }}$ is the input admittance. Insertion of (4) into (5) gives

$$
\frac{1-\rho_{1}}{1+\rho_{1}}=\frac{1-\rho_{2}}{1+\rho_{2}}+\frac{\pi}{2 Q_{r}}
$$

where we have introduced the reflections from the resonator ends without radiation, as seen from inside the resonator, defined by

$$
\begin{aligned}
& \rho_{1}=\frac{Y-Y_{0}^{\prime 2} / Y_{0}}{Y+Y_{0}^{\prime 2} / Y_{0}} \\
& \rho_{2}=\frac{Y-Y_{00}^{\prime 2} / Y_{00}}{Y+Y_{00}^{\prime 2} / Y_{00}} .
\end{aligned}
$$

2) The phase shift through the resonator is fixed to the same value for all elements in the array. With the purpose to calculate the phase shift through the resonator from reference plane $B$ to $C$ (Fig. 1), we recognize that at these planes the resonator is connected to equivalent input and output lines with the characteristic admittances $Y_{0}{ }^{2} / Y_{0}$ and $Y_{00}{ }^{2} / Y_{0}$, respectively, obtained by the transformation of the real transmission line characteristic admittances through the quarter wavelength transformers. The reflection coefficients from the resonator ends, when radiation is present, as seen from inside the resonator are then found to be

$$
\begin{aligned}
& \rho_{1}^{\prime}=\frac{Y-Y_{0}{ }^{\prime 2} / Y_{0}-G}{Y+Y_{0}{ }^{\prime 2} / Y_{0}+G} \\
& \rho_{2}^{\prime}=\frac{Y-Y_{00}{ }^{\prime 2} / Y_{00}-G}{Y+Y_{00}{ }^{\prime 2} / Y_{00}+G} .
\end{aligned}
$$

The field transmission coefficient from point $B$ to $B^{\prime}$ is given by

$$
\eta_{1}=1-\rho_{1}^{\prime}=\frac{2 Y_{0}{ }^{\prime 2} / Y_{0}}{Y_{0}^{\prime 2} / Y_{0}+Y+G} .
$$

Similarly, we find the field transmission coefficient from point $C^{\prime}$ to $C$ :

$$
\eta_{2}=1+\rho_{2}^{\prime}=\frac{2 Y}{Y_{0}{ }^{2} / Y_{0}+Y+G} .
$$

The transmitted electric field at $C$ is then related to the field in the incoming wave at $B$ through an expansion of multiple reflections in the resonator

$$
\begin{aligned}
E_{t} & =E_{i} \eta_{1} \eta_{2} \exp \left(-j \pi f / f_{c}\right) \sum_{n=0}^{\infty}\left[\rho_{1}^{\prime} \rho_{2}{ }^{\prime} \exp \left(-j 2 \pi f / f_{c}\right)\right]^{n} \\
& =E_{i} \frac{-\eta_{1} \eta_{2} \exp \left(-j \pi \Delta f / f_{c}\right)}{1-\rho_{1}{ }^{\prime} \rho_{2}{ }^{\prime} \exp \left(-j 2 \pi \Delta f / f_{c}\right)}
\end{aligned}
$$

where $f_{c}$ is the center frequencey and $\Delta f=f-f_{c}$.
The total phase-shift change through the resonator due to the frequency change $\Delta f$ is then found from (13)

$$
L\left(E_{t} / E_{i}\right)=-\pi \Delta f / f_{c}-\delta_{\text {res }}
$$

where the first term is due to the resonator length, and

$$
\delta_{\text {res }}=\arctan \frac{K \sin \left(2 \pi \Delta f / f_{c}\right)}{1-K \cos \left(2 \pi \Delta f / f_{c}\right)}
$$

is caused by the resonator effect. Here we have defined

$$
K=\rho_{1}{ }^{\prime} \rho_{2}{ }^{\prime}
$$

A more convenient expression for $K$, obtained by insertion of (9) and (10) in (16) and using the approximation $\left(G Z / 2 \rho_{1}\right)^{2} \ll 1$, is

$$
K \cong \rho_{1}^{2} \text {. }
$$

3) The division of the input power in radiated and transmitted power regulates the excitations of the elements and the array radiation pattern. The powers $P_{i}$ and $P_{t}$ in the incident wave at $B$ and output wave at $C$ are found as the product of the respective transformed transmission line characteristic admittance and the electric field squared. Hence we define the power transmission coefficient

$$
T=\frac{P_{t}}{P_{i}}=\left(\frac{Y_{00}{ }^{\prime 2}}{Y_{00}} E_{t}^{2}\right) /\left(\frac{Y_{0}^{\prime 2}}{Y_{0}} E_{i}^{2}\right)
$$

Since the resonator is matched at $f_{c}$ at the input, $E_{t} / E_{i}=-1$. Using (7) and (8) we then find

$$
T=\frac{1-\rho_{2}}{1+\rho_{2}} \cdot \frac{1+\rho_{1}}{1-\rho_{1}}
$$

With specified values of $K$ and $T,(6),(17)$, and (19) can be used to find $\rho_{1}, \rho_{2}$, and $Q_{r}$. With the aids of (1), (7), and (8), the characteristic admittances $Y, Y_{0}{ }^{\prime}$, and $Y_{00}{ }^{\prime}$ can be found. In Fig. 2 curves of $Z_{0}{ }^{\prime}=1 / Y_{0}{ }^{\prime}, Z_{00}=1 / Y_{00}$, and $Q_{r}$ versus $T$ for different values of $K$ are shown, with $h=0.304 \mathrm{~mm}$ and $\epsilon_{r}=2.4$.

A restriction to the performance of the element is given by the conductor and dielectric losses. The corresponding quality factor $Q_{0}$ is the highest possible $Q_{r}$ value applicable in the design procedure. In any case the efficiency of each element is $\eta_{e}=Q_{0} /\left(Q_{0}+Q_{r}\right)$

\section{FREQUENCY SCANNING ARRAY}

A frequency scanning array is formed by $N$ elements spaced $p$ freespace wavelengths and cascade-coupled by transmission lines of the length $L$ and transmission line wavelengths $\lambda_{g}$ (Fig. 3). The characteristics of the antenna are described on the basis of

1) the main beam scanning angle $\theta_{m}$,

2) the 3-dB beamwidth $\Delta \theta_{3 \mathrm{~dB}}$, and

3) the sidelobe level. 


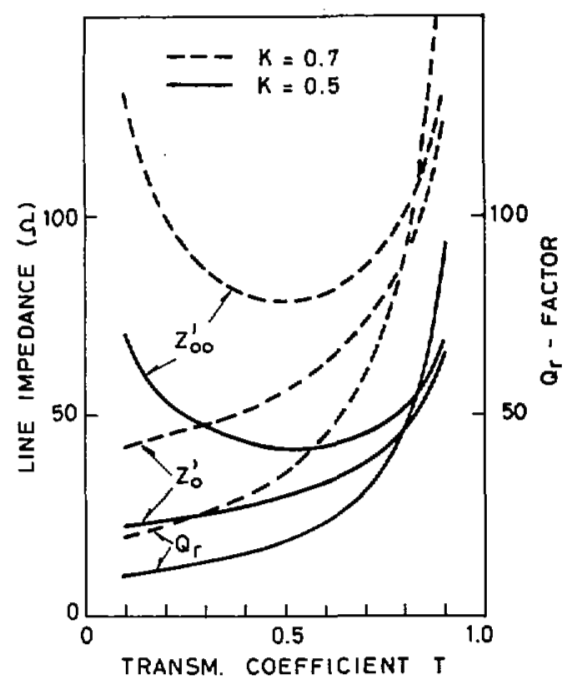

Fig. 2. Characteristic impedances $Z_{0}{ }^{\prime}$ and $Z_{00}{ }^{\prime}$ for input and ouput transformers, respectively, and radiation $Q_{r}$ versus transmission factor $T$ with phase factors $K=0.5$ and 0.7 .

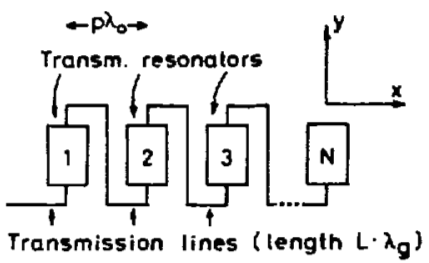

(a)

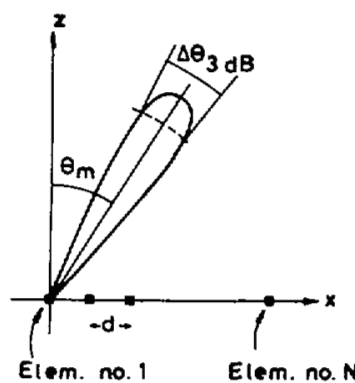

(b)

Fig. 3. (a) Structure of antenna array. (b) Quantities characterizing antenna array.

The possibilities of forming different radiation patterns are limited in the present antenna geometry by a technologically determined minimum width $(0.1 \mathrm{~mm})$ of the microstrip used in the $\lambda / 4$-transformer couplings.

An array well suited for examination of the frequencyscanning characteristics, because of its simple structure, is the antenna with identical elements, i.e., equal $T$ for the elements, giving an exponential decay of the excitation. An exception is the last $\left(N^{\prime}\right.$ th) element in the array, which is matched and hence has a different width, which is found from (6), setting $\rho_{2}=1$, and (1) and (7).

This array is also simple to fabricate, using a photographic repetition technique. The tolerance for each element is the same, giving only a frequency shift for the antenna and almost no disturbance of the other characteristics.

As a consequence of the exponential variation of the element excitation, $\Delta \theta_{3} \mathrm{~dB}$ has an optimal value when the number of elements $N$ exceeds a certain value, determined by $T$ and the element-to-element field attenuation factor

$$
\gamma=10^{-\frac{\alpha L^{\prime} \lambda_{g}}{20}}
$$

where $\alpha(\mathrm{dB} / \mathrm{m})$ is the attenuation probability unit length of the transmission line.

Anticipating a power input to the first element of $1 \mathrm{~W}$ the power input to the $m$ th element is $\left(T \gamma^{2}\right)^{m-1}$. The fraction $(1-T)$ of this power radiates. The $N$ th element matches the array at the end. Hence the radiated power from the $m$ th element is

$$
P_{m}= \begin{cases}(1-T)\left(T \gamma^{2}\right)^{m-1}, & 1 \leqslant m \leqslant N-1 \\ \left(T \gamma^{2}\right)^{N-1}, & m=N .\end{cases}
$$

In this expression we neglected the mutual coupling between the elements. The excitation of the $m$ th element is then

$$
I_{m}=I_{0} \sqrt{P_{m}}, \quad 1 \leqslant m \leqslant N
$$

where $I_{0}$ is the normalization constant.

The radiation pattern with equispaced elements situated on the $X$-axis (Fig. 3(b)) is

$$
G(v)=\sum_{m=1}^{N} I_{m} \exp (-j(m-1) v)
$$

where

$$
v=\delta+k d \sin \theta
$$

and $k$ is the propagation factor, $d=p \lambda$ and $\delta$ are the elementto-element distance and phase-shift, respectively, and $\theta$ is the angle between the beam and broadside direction. After performing the summation of (23) and insertion of (21) and (22), we find

$$
\begin{aligned}
G(v(\theta))= & I_{0} \sqrt{1-T} \frac{1-\left(\gamma \sqrt{T} e^{-j v}\right)^{N-1}}{1-\gamma \sqrt{T} e^{-j v}} \\
& +I_{0}\left(\gamma \sqrt{T} e^{-j v}\right)^{N-1}
\end{aligned}
$$

$|G(v)|^{2}$ forms the $v$-curve of the radiation pattern, from which we find $v=v_{3} \mathrm{~dB}$, where $|G|^{2}$ has decreased $3 \mathrm{~dB}$. Using (24) we then find $\Delta \theta_{3 \mathrm{~dB}}$ by numerical calculations.

In the simple and optimal case, where the number of elements $N \gg 1 /(\gamma \sqrt{T})$, we find

$$
\Delta \theta_{3 \mathrm{~d} \mathbf{B}} \approx(1-\gamma \sqrt{T}) /(\pi p)
$$

The scanning angle is found to be

$$
\theta_{m}=\arcsin \left(-\frac{\delta}{2 \pi p}\right)
$$

Here $\delta$ is given by

$$
\delta=\delta_{\text {res }}+2 \pi \frac{\Delta f}{f_{c}}(L+1)
$$


where $\delta_{\text {res }}$, given by (15), accounts for the phase-shift due to the resonator effect and the second term accounts for the phase-shift due to the transmission line length $(L \cdot \lambda)$ and the transformer plus resonator length $(\lambda)$.

In the design of the array we must avoid grating lobes for all frequency shifts $\Delta f$ lower than the maximum shift $\Delta f_{\max }$. This is obtained when the maximum value of $v$ is somewhat less than $2 \pi$, in practice determined to $v_{\max } \approx 300^{\circ}$. According to (24), $v=v_{\max }$ when $\theta=\pi / 2$, and $\delta=\delta_{\max }$. Hence we find

$$
v_{\max }=\delta_{\max }+2 \pi p .
$$

An additional design equation is obtained from (27), for the maximum beam scanning angle $\theta_{m}=-\theta_{\text {max }}$ :

$$
0=\delta_{\max }-2 \pi p \sin \theta_{\max } .
$$

In the design prescribed values of $\theta_{\max }$ and $v_{\max }$ inserted into (29) and (30) result in solutions of $\delta_{\max }$ and $p$. The phase-shift $\delta_{\max }$ is, according to (28), obtained by selection of $\delta_{\text {res }}, \Delta f_{\max }$, and $L$ to suitable values. $\delta_{\text {res }}$ and $\Delta f_{\max }$ are limited by the resonator $K$ value and bandwidth. $L$ can be varied up to a limit determined by $\Delta \theta_{3 \mathrm{~dB}}$ and $\gamma$ is given by (26) and (20). In the situations requiring small $\Delta \theta_{3 \mathrm{~dB}}$ and large $\theta_{m}$, a trade-off between these quantities has to be done. In many situations the overall efficiency

$$
\eta=\eta_{e}(1-T) /\left(1-T \gamma^{2}\right)
$$

has to be taken into account, where $\eta_{e}$ is defined in Section II.

Typically, we find that with the parameters $L=5, T=$ $0.9, \gamma=0.95, K=0.65$, and $f_{c}=9.8 \mathrm{GHz}$, the optimum $N=40$, and with $\theta_{\max }=45^{\circ}, \Delta \theta_{3 \mathrm{~dB}}=5.7^{\circ}, \Delta f=450 \mathrm{MHz}$, and $\eta / \eta_{e}=0.7$.

\section{EXPERIMENTS}

Two antennas have been constructed to verify the theory. For both antennas $N=19, p=0.406, L=4, h=0.304 \mathrm{~mm}$, and $T=0.9$. The phase parameters were $K=0.5$ and 0.65 for the two antennas. The geometry is shown in Fig. 3.

Radiation patterns were recorded in the radio anechoic chamber at the Electromagnetics Institute. In the $y z$-plane the pattern varies about $6 \mathrm{~dB}$ from the direction perpendicular to parallel to the antenna plane in accordance with the singleelement pattern described in [12]. In the $x z$-plane patterns were measured at frequencies from $9-10.5 \mathrm{GHz}$ with intervals of $100 \mathrm{MHz}$. Fig. 4 shows some of these diagrams for the antenna with $K=0.65$. The pattern at the center frequency $9.6 \mathrm{GHz}$ is shown to be in good agreement with the theory, regarding the sidelobe level, while the experimental beamwidth is 10-20 percent larger than the theoretical value (Fig. 5).

The reason might be overestimated values for $\gamma$ and $T$. Unwanted reflections caused by a mismatch of the resonators can also be a reason, since they will add to the excitations with different phase from element to element. Such reflections will also make the minima between the sidelobes less distinct than predicted. The reflections increase at frequencies far from the center frequency, which causes a wider beamwidth and higher sidelobe level. Hence the bandwidth of the resonators limits the usable frequency interval.

Apart from an offset error of the center frequency, due to a 3 percent ertor in the layout, the measured and calculated

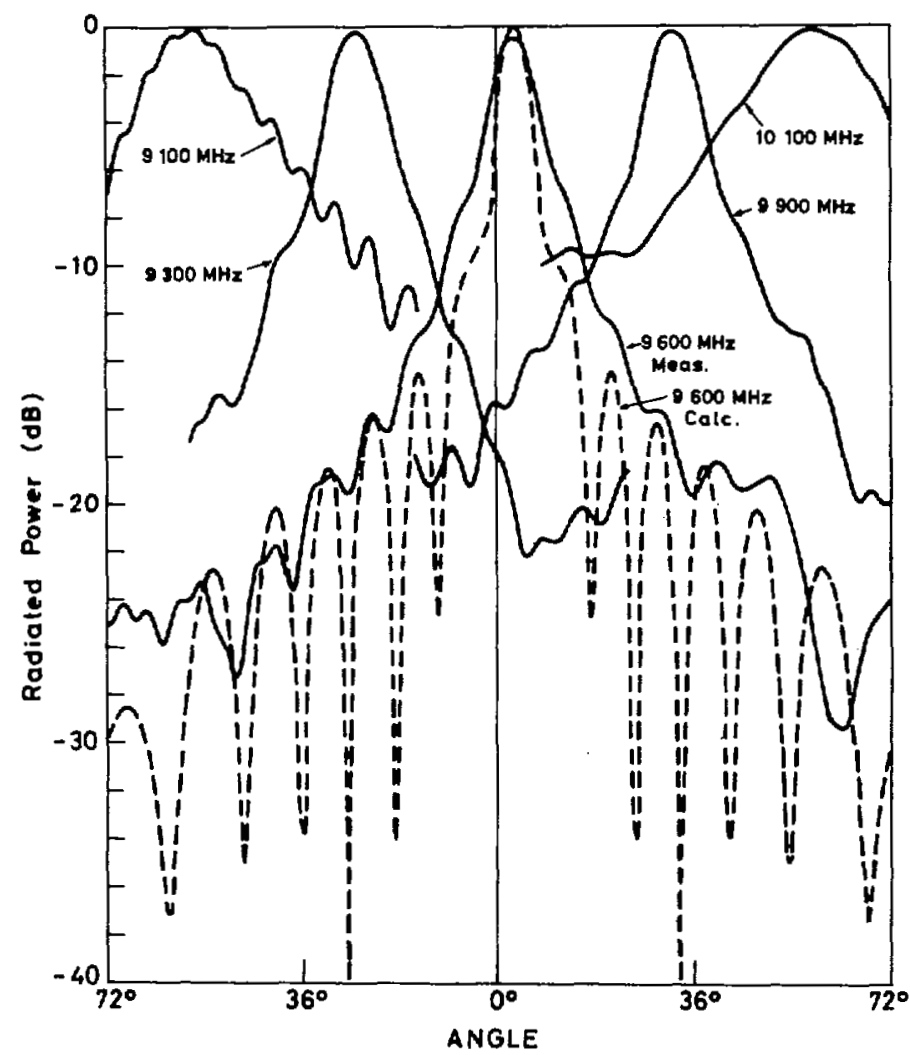

Fig. 4. Radiation patterns for antennas with parameters $T=0.9$, $K=0.65, \gamma=0.95$. Full lines: measured patterns. Dotted line: theoretical pattern for $9600 \mathrm{MHz}$.

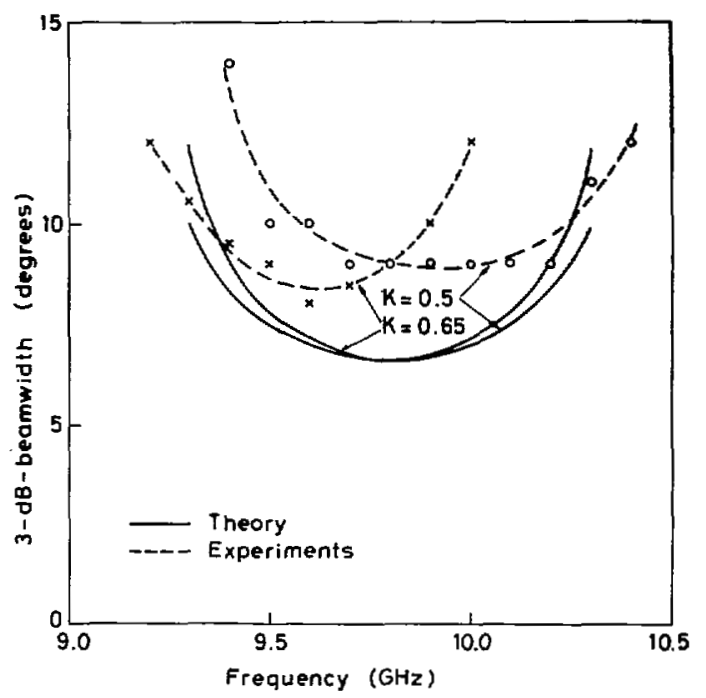

Fig. 5. 3-dB-beamwidth versus frequency for antennas with phase factor $K=0.5$ and 0.65 , respectively. For both antennas $T=0.9$ and $\gamma=0.95$.

dependences of the main beam direction $\theta_{m}$ on the frequency are in excellent agreement (Fig. 6) for both antennas.

In Table I we have compared the theoretical and measured phase-shifts through the resonators for a frequency shift between points in Fig. 5 corresponding to a beamwidth of $\Delta \theta_{3 \mathrm{~dB}}=10 \mathrm{deg}$. The corresponding scanning angle $\theta_{\mathrm{m}}$, found from Fig. 6, and (27) give a measured phase-shift $\delta$. The measured $\delta_{\text {res }}$ is found by subtracting the phase-shift caused by the line length from $\delta$. Comparison of this value 


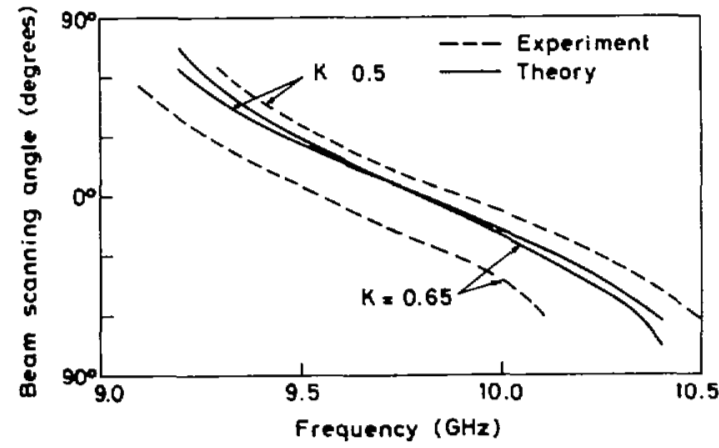

Fig. 6. Main beam direction versus frequency for antennas with phase factor $K=0.5$ and 0.65 , respectively. For both antennas $T=0.9$ and $\gamma=0.95$.

TABLE I

BEAM SCAN AND CHANGES IN PHASE-SHIFT THROUGH SINGLE RESONATORS BETWEEN FREQUENCIES WHERE $\Delta \theta_{3} \mathrm{~dB}=$ 10 DEG

\begin{tabular}{|c|c|c|c|c|c|c|}
\hline $\begin{array}{l}\text { Antenna } \\
\text { with } \\
K=\end{array}$ & $\begin{array}{c}\text { Minimum } \\
\text { frequency } \\
\text { [GHz] }\end{array}$ & $\begin{array}{c}\text { Maximum } \\
\text { frequency } \\
\text { [GHz] }\end{array}$ & $\begin{array}{c}\text { Beam-scar } \\
\theta_{\mathrm{m}} \\
\text { (degrees) }\end{array}$ & $\begin{array}{l}\text { Tolal } \\
\text { phose-shift } \\
\text { d (degrees) }\end{array}$ & $\begin{array}{l}\text { Resonator } \\
\text { Measured } \\
\text { (degrees) }\end{array}$ & $\begin{array}{l}\text { phase-shill } \delta_{\text {ras }} \\
\text { |Colculated } \\
\text { (degrees) }\end{array}$ \\
\hline 0.5 & 9.6 & 10.2 & \pm 25 & 61 & 6 & 11 \\
\hline 0.65 & 9.25 & 9.85 & .30 & 73 & 18 & 19 \\
\hline
\end{tabular}

with the theoretical one found from (15) shows that the resonator effect contributes up to 25 percent total phase-shift for the antennas.

The directivity found from integration of the theoretical pattern is $15.9 \mathrm{~dB}$. The directivity of the experimental antenna is estimated to be $14.7 \mathrm{~dB}$ on the basis of the measured radiation pattern. The gain was measured to be $8.1 \mathrm{~dB}$ for the antenna with $K=0.5$. The difference of $-6.6 \mathrm{~dB}$ is somewhat larger than the theoretically estimated efficiency of $-5 \mathrm{~dB}$, and can be interpreted by the somewhat higher ohmic loss than used for the theoretical estimation. Such a higher loss will also result in a lower $\gamma \sqrt{T}$ product, i.e., lower effective antenna length and hence a lower experimental than theoretical directivity, as actually was found.

\section{CONCLUSION}

Investigation of frequency scanning microstrip antennas has shown that a beam scan of $\theta_{m}= \pm 30$ degrees with a 3-dB beamwidth less than 10 degrees is possible using a frequency sweep interval of $\pm 300 \mathrm{MHz}$. Scanning angles up to \pm 45 degrees with some degradation of the beamwidth can be obtained. The sidelobe level is $-12 \mathrm{~dB}$. Increasing the element number to the optimum would decrease the beamwidth by 50 percent.

A drawback to the applicability of frequency-scanning antennas is the losses in the microstrip. The efficiency might be improved by using a thicker substrate and making a tradeoff between the efficiency and scanning angle.

A restriction for the antennas is also the minimum line width of the coupling transformers of $0.1 \mathrm{~mm}$, set by the photo lithographic process. This results in maximum possible values of $K \cong 0.7$ and $T=0.9-0.95$. It is suggested that capacitive coupling to the elements could extend the ranges of $K$ to $0.6-1.0$ and of $T$ to $0.9-1.0$.

\section{APPENDIX}

The integral $F_{1}{ }^{\prime}(\epsilon, n)$ from [13], where $\epsilon$ is the effective dielectric constant and $n$ is the number of half wavelengths in the resonator, can be written

$$
\begin{aligned}
F_{1}^{\prime}(\epsilon, n)= & 2 F_{1}(\epsilon)+\frac{(\epsilon-1)^{2}}{\epsilon \sqrt{\epsilon}}\left[\mathrm{Ci}\left(n \pi \frac{\sqrt{\epsilon}+1}{\sqrt{\epsilon}}\right)\right. \\
& -\mathrm{Ci}\left(n \pi \frac{\sqrt{\epsilon}-1}{\sqrt{\epsilon}}\right)+n \pi \mathrm{Si}\left(n \pi \frac{\sqrt{\epsilon}+1}{\sqrt{\epsilon}}\right) \\
& \left.-n \pi \mathrm{Si}\left(n \pi \frac{\sqrt{\epsilon}-1}{\sqrt{\epsilon}}\right)\right] \\
& -(-1)^{n}\left(\frac{4}{n \pi \sqrt{\epsilon}} \sin \frac{n \pi}{\sqrt{\epsilon}}+2 \frac{\epsilon-1}{\epsilon} \cos \frac{n \pi}{\sqrt{\epsilon}}\right)
\end{aligned}
$$

where

$$
F_{1}(\epsilon)=\frac{\epsilon+1}{\epsilon}-\frac{(\epsilon-1)^{2}}{2 \epsilon \sqrt{\epsilon}} \ln \frac{\sqrt{\epsilon}+1}{\sqrt{\epsilon}-1}
$$

is the function for the radiation from an open-circuit microstrip [12]. The first term in (A1) gives the radiation from the resonator where no mutual coupling is between the two ends, and the remaining terms account for mutual coupling. These terms cancel in the cases when $\epsilon=1$ and for $n \rightarrow \infty$, taking the asymptotic expansion of $\operatorname{Si}(\alpha)$ into consideration.

\section{REFERENCES}

[1] R. E. Munson, "Conformal microstrip antennas and microstrip phased arrays," IEEE Trans. Antennas Propagat., vol. AP-22, pp. 74-78, Jan. 1974.

[2] J. Q. Howell, "Microstrip antennas" IEEE Group on Antennas and Propagation Int. Symp., p. 177, Dec. 1972.

[3] G. G. Sanford, "Conformal microstrip phased array for aircraft tests with ATS-6," National Electronics Conf., vol. 29, pp. 252-257, Oct. 1974.

[4] J. Q. Howell, "Microstrip antennas" IEEE Trans. Antennas Propagat. vol. AP-23, pp. 90-93, Jan. 1975.

[5] C. W. Garvin et al., "Low-profile electrically small missile base mounted microstrip antennas," IEEE Society on Antennas and Propagation Int. Symp., pp. 244-247, June 1975.

[6] G. Sanford and L. Klein, "Development and test of a conformal microstrip airborne phased array for use with the STS-6 satellite," IEEE Int. Conf. on Antennas for Aircraft and Spacecraft, pp. 115-122, June 1975.

[7] J. R. James and G. J. Wilson, "New design techniques for microstrip antenna arrays," 5th European Micrawave Conf., pp. 102106, Sept. 1975.

[8] F. R. Zboril, "New antennas on new nonmetallic materials," 5th European Microwave Conf., pp. 658-662, Sept. 1975.

[9] A. G. Derneryd, "Linearly polarized microstrip antennas," IEEE Trans. Antennas Propagat., vol AP-24, pp. 846-851, Nov. 1976.

[10] R. C. Hansen, Microwave Scanning Antennas, vol. III. New York: Academic, 1966.

[11] M. Tiuri, J. Henriksen, and S. Tallquist, "Printed circuit radio link antenna," 6th European Microwave Conf., pp. 280-283, Rome, Sept. 1976.

[12] L. Lewin, "Radiation from discontinuities in strip-line," Inst. Elec. Eng., Monograph 1358E, pp. 163-170, Feb. 1960.

[13] J. Watkins, "Radiation from open-circuited dielectric resonators," IEEE Trans. Microwave Theory Tech., vol. MTT-21, pp. 636-640, Oct. 1973.

[14] Belahubek and Denlinger, "Loss considerations for microstrip Iesonators", IEEE Trans. Microwave Theory Tech., vol. MTT23, pp. 522-526, June 1975.

[15] H. A. Wheeler, "Transmission-line properties of parallel strips separated by a dielectric sheet," IEEE Trans. on Microwave Theory Tech., vol. MTT-13, p. 172, Mar. 1965. 